\title{
Other BSM Searches at the LHC
}

\author{
Kamal Benslama* on behalf of the ATLAS and CMS collaborations \\ University of Regina, Canada \\ E-mail: kamal.benslama@gmail.com
}

The considerable center-of-mass energy and luminosity provided by the Large Hadron Collider (LHC) will ensure a discovery reach for new particles which extends well into the multi-TeV region. ATLAS and CMS have carried out many studies of the implications of this capability for Beyond the Standard Model physics. In this paper, we summarize the results of studies involving, leptons and jets with and without missing transverse energy in the final state. We will mainly focus on the potential for discovery and limits setting with an integrated luminosity of the order of 100 to $200 \mathrm{pb}^{-1}$ per experiment.

XXth Hadron Collider Physics Symposium

November 16 - 20, 2009

Evian, France

* Speaker. 


\section{Introduction}

The ATLAS [1] and CMS [2] experiments at the LHC will give access to an unprecedented range of processes at $p_{T}$ scales and event rates far in excess of those generated by earlier colliders. Many rare Standard Model (SM) processes will be studied with high statistics, while at the same time searches will be carried out for particles predicted to exist by theories extending the SM. The need to stabilise the mechanism for breaking electroweak (EW) symmetry in the SM requires that at least some of these new particles have masses of the order of the EW scale $(\sim 100 \mathrm{GeV})$. Hence, there is every reason to believe that they should be observable at the LHC.

In this paper we review the results of some of the studies of Beyond the Standard Model physics at the LHC which have been carried out by ATLAS and CMS, concentrating on those involving leptons and jets with and without missing transverse energy in the final state. For reasons of space, we will mainly focus on the potential for discovery and limits setting with an integrated luminosity of the order of 100 to $200 \mathrm{pb}^{-1}$ per experiment.

\section{Dileptons searches}

In several models beyond the Standard Model (BSM), final states with dileptons can provide striking evidence of new high mass resonances in a relatively clean search. Both the ATLAS and CMS experiments have good mass resolutions which will allow these objects to be reconstructed up to momenta of the order of $1 \mathrm{TeV}$.

These searches look for two well reconstructed leptons of the same flavor and opposite charges, both with $|\eta|<2.5$ (where $\eta$ is the pseudorapidity of the lepton). No requirement is imposed in the dilepton opening angle. Given the large rejection factors that both experiments expect against QCD backgrounds, the main background for this type of search is expected to be the Drell-Yan production. In what follows, we will refer to the SSM model (in which $Z^{\prime}$ couplings are the same as those of the SM Z boson), unless otherwise noted.

\subsection{Background determination}

Since detector effects and theoretical uncertainties can affect the Monte Carlo based background estimation, both collaborations are developing control sample strategies to help constrain some of the expected backgrounds. Figure 1 shows, for example, an estimation of the $t \bar{t}$ background from the number of electron-muon events, since they can be expected to be twice as numerous as the dielectrons events (from this process). The $t \bar{t}$ spectrum determined using this method (black triangles) is compared with the Monte Carlo $t \bar{t} \rightarrow e e$ distribution (solid yellow histogram), showing good agreement [3].

\subsection{Physics reach}

The discovery potential has been estimated using several methods, all with compatible results. In one of them, the generator-level shape of the dilepton invariant mass distribution was modeled with a four-parameter family of curves; these parameters describe the mass and the width of the $Z^{\prime}$ resonance, its amplitude and the size of its interference with the tail of the SM Z boson. Detector 


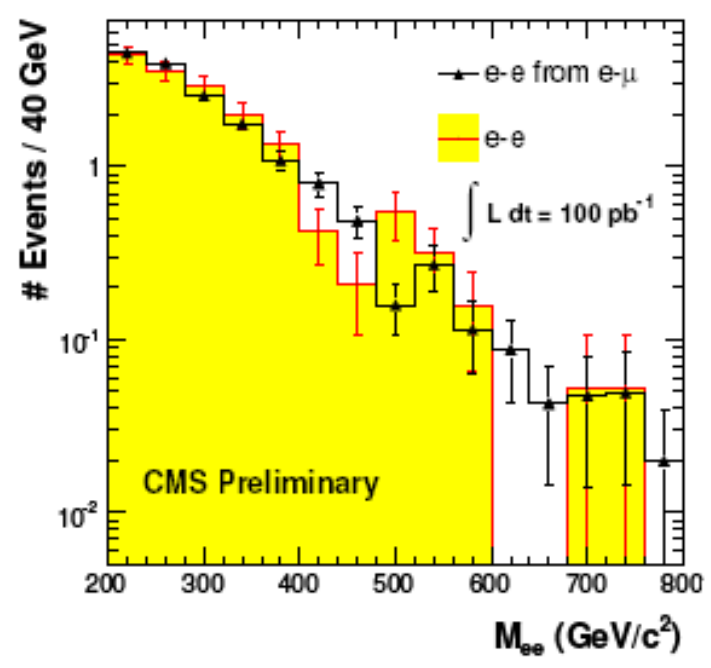

Figure 1: $t \bar{t}$ background estimation from $e \mu$ combinations (triangles); histogram: MC truth.

resolution, acceptance effects and selection efficiencies were obtained from full simulation samples, and applied to these shapes. From these models, the expected sensitivity is obtained through the use of the log-likelihood ratio (LLR) estimator, in which the Background only (null hypothesis, H0) distribution is compared with the Signal+Background (H1) distribution to compute the probability that, when no signal is present, an outcome will have a peak at least as large as the expected signal. This is done by comparing the expected distribution of the LLR for each hypothesis [4]. Using this procedure, there is no need to restrict the analysis to a mass window. Both experiments have comparable physics reach in these searches. Figure 2 shows the expected luminosity needed to reach a statistical significance of $5 \sigma$ for several $Z^{\prime}$ models [4]. If such a state exists slightly above the current Tevatron limits (1 TeV [5] ), as little as $100 \mathrm{pb}^{-1}$ of integrated luminosity could yield a $5 \sigma$ discovery even for the least favorable model considered $\left(Z_{\psi}^{\prime}\right)$. Both collaborations have assessed the effect of lowering the center of mass energy; in particular, when going from 14 to 10 $\mathrm{TeV}$; the production cross-sections for signal and backgrounds are reduced by factors of 2 or 3 (for masses of the $Z^{\prime}$ boson between 1 and $2 \mathrm{TeV}$ ); accordingly, the luminosity needed for discovery roughly doubles [6]. Besides $Z^{\prime}$ models, the physics reach for other possible extensions of the SM has also been evaluated. As an example, the sensitivity of a dimuon search for the Graviton in Randall-Sundrum models is illustrated in Figure 2 [7].

\section{Leptons plus jets searches}

Final states with two leptons and one or two jets also have a good chance of allowing the LHC to improve the current experimental limits at the early stages of data taking. Both leptoquark (LQ) models and Left-Right Symmetric Models (LRSM) produce this signature, and in both cases the background can be reduced strongly. 

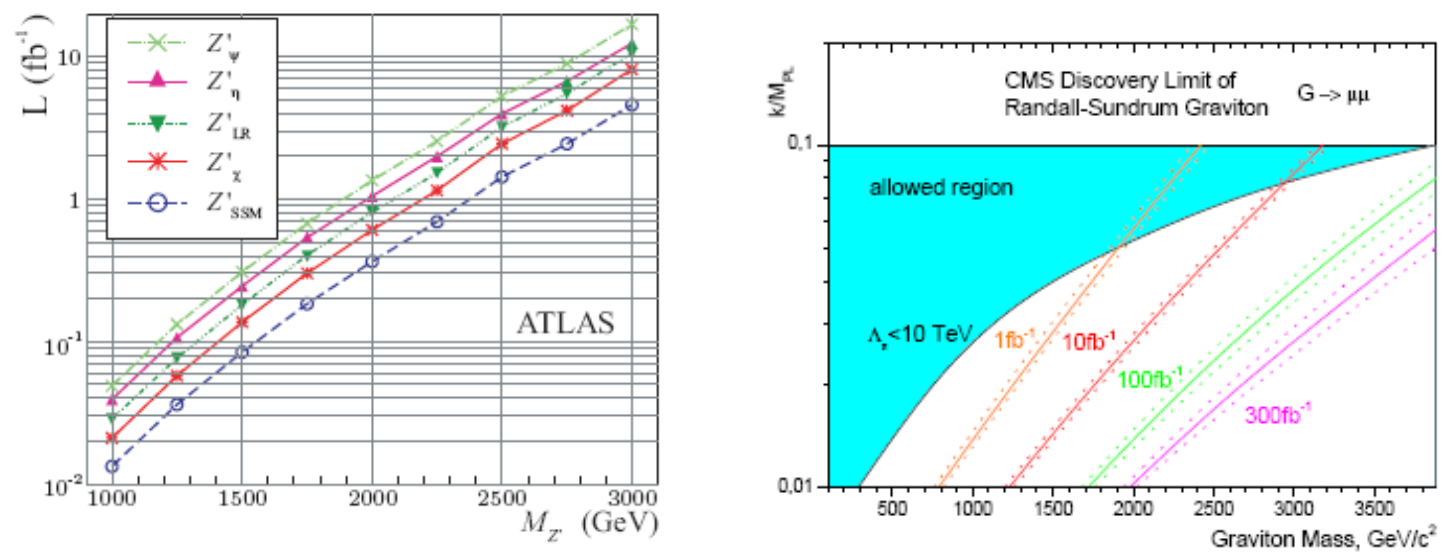

Figure 2: Left: Expected luminosity needed for $5 \sigma$ discovery for several models of the $Z^{\prime}$ boson, as a function of the $Z^{\prime}$ mass. Right: Discovery reach of the dimuon search for Randall-Sundrum gravitons as a function of the coupling $k / M_{P L}$ and the graviton mass. For each luminosity shown, the expected significance exceeds $5 \sigma$ in the region to the left of the corresponding curve. Dotted curves show expected variations due to systematic uncertainties.

\subsection{Leptoquarks}

Among possible new particles in physics beyond the SM, leptoquarks (LQs) are an interesting category of exotic color triplets with coupling to quarks and leptons. They are a generic prediction of Grand Unified Theories [8], of composite models [9], of technicolor schemes [10], of superstring-inspired $E_{6}$ models [11], and of supersymmetry with R-parity violation [12]. Current experimental limits (from D0) place the mass of the first-generation LQ over $256 \mathrm{GeV}$, and that of the second generation LQ above $251 \mathrm{GeV}$. For the search, events are required to have 2 leptons of opposite charge and the same flavor and at least two jets. Expected backgrounds (Drell-Yan, $t \bar{t}$ and diboson production) are rejected by requirements on the transverse momenta of the leptons, the scalar sum of the $p_{T}$ of leptons and jets, the dilepton invariant mass and the lepton-jet invariant mass. Figure 3 shows the $5 \sigma$ contours for the leptoquark search in ATLAS, showing the branching ratio $(\beta)$ that would yield a $5 \sigma$ discovery as a function of the total integrated luminosity used, for a $400 \mathrm{GeV}$ leptoquark [4]. For this mass, a value of $\beta^{2}$ above 0.3 would allow a $5 \sigma$ signal with less than $100 \mathrm{pb}^{-1}$ of data.

\subsection{Left-right symmetric models}

These models incorporate three heavy right-handed Majorana neutrinos $\left(N_{e}, N_{\mu}, N_{\tau}\right)$, and some also introduce right-handed heavy bosons $\left(W_{R}\right.$ and $\left.Z^{\prime}\right)$. In one of these models, the $W_{R}$ boson decays into a lepton and a Majorana neutrino $N_{l}$, which ultimately produces a lepton and two jets. Since all final state particles can be reconstructed, it is possible in this model to reconstruct both the $N_{l}$ and the $W_{R}$ masses. Selection cuts similar to those used in the LQ searches allow a strong reduction of the expected backgrounds; Figure 3 [4] shows the expected significance of this search for two different scenarios; triangles correspond to the LRSM_18_3 scenario (defined by $m_{W_{R}}=1.8 \mathrm{TeV}$ and $m_{N_{e}}=m_{N_{\mu}}=300 \mathrm{GeV}$ ), while squares represent the LRSM_15_5 scenario (defined by $m_{W_{R}}=$ 

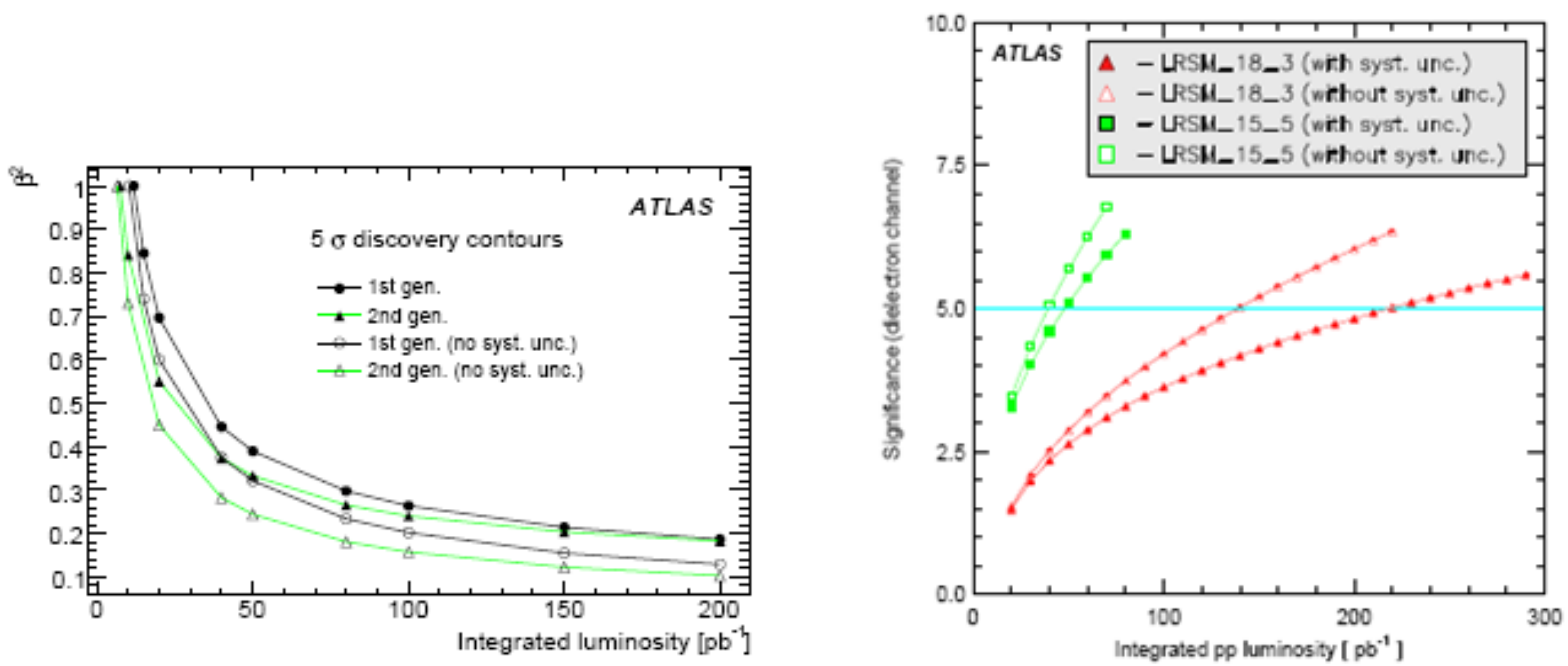

Figure 3: Left: $5 \sigma$ discovery potential for first and second generation leptoquarks (with $m_{L Q}=400 G e V$ ). Right: Expected signal luminosity for two mass hypotheses for the $N_{e}$ neutrino and the $W_{R}$ boson.

$1.5 \mathrm{TeV}$ and $\left.m_{N_{e}}=m_{N_{\mu}}=500 \mathrm{GeV}\right)$. Solid/hollow markers show the significance when systematic uncertainties are included/excluded. As shown, for these mass values, a $5 \sigma$ significance could be reached with less than $150 \mathrm{pb}^{-1}$ and $40 \mathrm{pb}^{-1}$ of collision data, respectively.

\section{Lepton-neutrino searches}

Studies involving a high $\mathrm{p}_{T}$ lepton and neutrino in the final state, as in the case of the standard model $\mathrm{W}$ boson, use the transverse mass as a discriminating variable. Events are required to have exactly, one isolated lepton (electron or muon), a large missing transverse energy and most of their missing transverse energy should come from the lepton and neutrino (i.e., they should have low jet activity). These cuts are effective to reduce the dijets and $t \bar{t}$ background. After applying these cuts, the main remaining background comes from the high $-m_{T}$ tail of the SM W boson. Figure 4 shows the $m_{T}$ distribution of three $m_{W^{\prime}}$ signals and the background after all cuts (electron channel) [14]; background distributions are stacked.

Figure 5 shows the expected luminosity needed to reach a $3 \sigma$ and a $5 \sigma$ evidence, as a function of the mass of the $W^{\prime}$ boson (using, as a benchmark, the Altarelli model [15] ), and the luminosity needed to set a $95 \%$ confidence level exclusion, also as a function of $\mathrm{m}\left(W^{\prime}\right)$. Due to the large production cross section of this model, an integrated luminosity of the order of $200 \mathrm{pb}^{-1}$ is expected to allow LHC experiments to probe masses up to about $2.5 \mathrm{TeV}$, which would be well above the current Tevatron limit of $1 \mathrm{TeV}$ [16].

\section{Summary}

With early data, and after a good understanding of the detectors in situ in the LHC environment, both ATLAS and CMS will be able to explore the energy regime beyond that of the Tevatron 


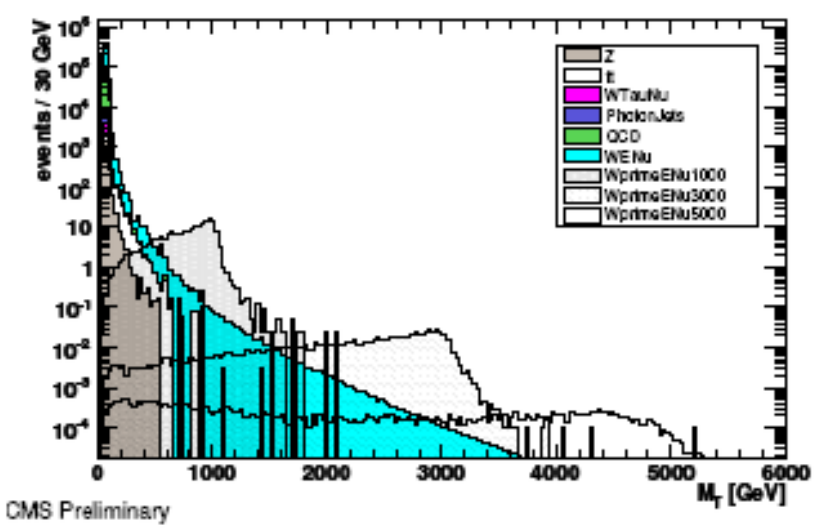

Figure 4: Transverse mass distribution after cuts [14] of signal and SM backgrounds in the $W^{\prime}$ search.
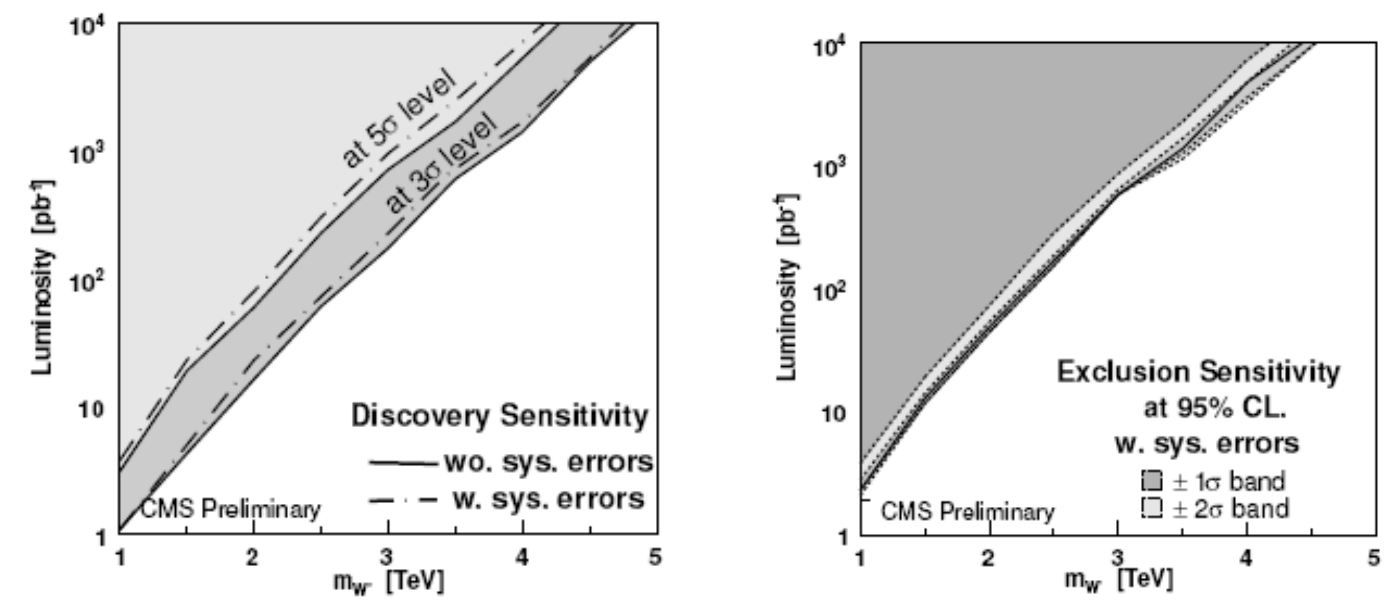

Figure 5: Left: Luminosity needed to establish evidence, as a function of the mass of $W^{\prime}$. Right: Luminosity needed to set $95 \%$ CL limit as a function of the mass of $W^{\prime}$.

with low integrated luminosities. With more data and more time, the LHC will explore the TeV scale in detail with direct discovery potential up to masses at the order of 5 to $6 \mathrm{TeV}$.

\section{References}

[1] The ATLAS Collaboration, JINST 3:S08003,2008.

[2] The CMS Collaboration, JINST 3:308004,2008.

[3] The CMS Collaboration, CMS-PAS-EXO-08-001.

[4] The ATLAS Collaboration, CERN-OPEN-2008-20.

[5] The CDF Collaboration, Phys. Rev. Lett 102, 091805, 2009. 
[6] The CMS Collaboration, CMS PAS EXO-09-006.

[7] The CMS Collaboration, CERN/LHCC 2006-021, CMS TDR 8.2 (2006).

[8] J. C. Pati and A. Salam, Phys. Rev. D 10 (1974) 275.

[9] B. Schrempp and F. Scrempp, Phys. Lett. B 153 (1985) 101.

[10] S. Dimopoulos and L. Susskind, Nucl. Phys B155 (1979) 237.

[11] V. D. Angelopoulos et al., Nucl. Phys. B 292 (1987) 59.

[12] A. F. Zarnecki, Eur. Phys. J. C 17 (2000) 695.

[13] V. A, Mitsou, et al., ATL-PHYS-CONF-2006-008, ATL-COM-PHYS-2004-071.

[14] The CMS Collaboration, CMS-PAS-EXO-08-004.

[15] G. Altarelli, B. Mele and M. Ruiz-Altaba, Z. Phys. C 45 (1989) 109 [Erratum-ibid. C 47 (1990) 676].

[16] V. M. Abazov et al. [D0 Collaboration], Phys. Rev. Lett. 100 (2008) 031804. 